\title{
ARCHITECTURE OF A SOFTWARE QUENCH MANAGEMENT SYSTEM
}

\author{
J.M.Nogiec, E.Desavouret, M.Lamm, D.Orris, S.Sharonov, J.Sim, \\ Fermi National Accelerator Laboratory, Batavia, IL 60510, USA
}

\begin{abstract}
Testing superconducting accelerator magnets is inherently coupled with the proper handling of quenches; i.e., protecting the magnet and characterizing the quench process. Therefore, software implementations must include elements of both data acquisition and real-time controls. The architecture of the quench management software developed at Fermilab's Magnet Test Facility is described. This system consists of quench detection, quench protection, and quench characterization components that execute concurrently in a distributed system. Collaboration between the elements of quench detection, quench characterization and current control are discussed, together with a schema of distributed saving of various quench-related data. Solutions to synchronization and reliability in such a distributed quench system are also presented.
\end{abstract}

\section{INTRODUCTION}

$\mathrm{R} \& \mathrm{D}$ programs of superconducting magnets require quench management systems that not only protect magnets but also supply the user with sufficient information to perform quench analysis. Important test conditions have to be recorded (such as temperatures, flows, current, and pressures) as well as data about quench origin, quench propagation, and strains. The system should be also configurable to adjust to differently instrumented magnets of various types.

\section{FRAMEWORK}

To promote reuse, reduce the complexity of development, and allow for easy exchange of information, the quench management system has been built on top of a framework.

The framework used is a configurable and extensible system; it has been designed to be a foundation for building distributed monitoring and control systems [1][2].

The configuration of the system is described in a specifically designed language. With this language the user can specify: tasks that input and output data (scans), process variables and their associations with DAQ hardware, sensors, and calculations. The configured system is distributed among several computing nodes. Each node has a memory-resident Real-Time Data Base (RTDB), which contains both configuration and acquired data. RTDB is accessible remotely through a server task.

Scans communicate and synchronize their actions via the exchange of events over a distributed software bus. An event is delivered only to those components that have previously registered interest (subscribed) in this event. A set of programmatic interfaces is provided to enable remote and local access to data, configuration, and the event bus. A set of GUIs is also provided, to facilitate monitoring and control of the system (scan control panel, event monitor, main system management panel, system status display) as well as to enable data visualization (configurable numerical display, and configurable charting display).

\section{ARCHITECTURE}

The quench system consists of several cooperating subsystems: quench detection, quench protection, quench characterization, quench management, and power supply control. These subsystems are distributed in several nodes (see Fig. 1) and communicate through the software event bus and/or hardware signals.

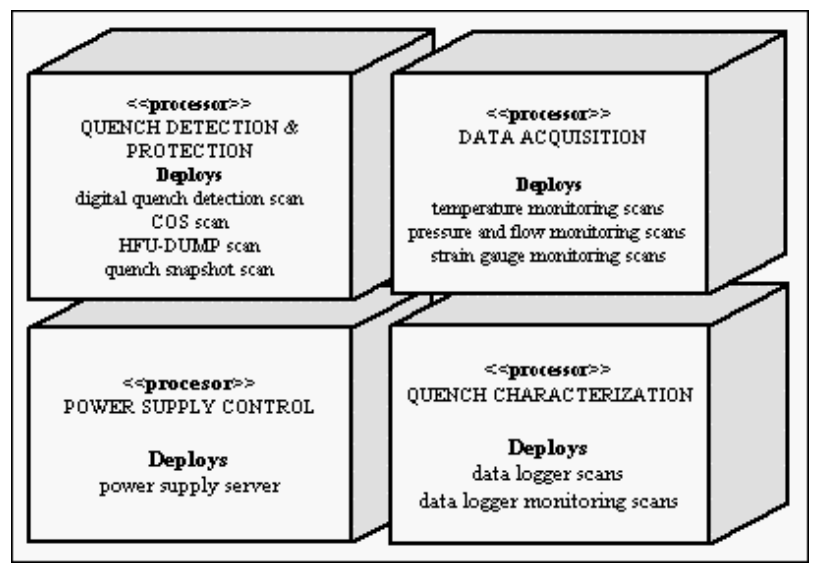

Figure 1: Quench system deployment diagram.

\subsection{Quench Detection}

Quenches can be detected through monitoring of coil voltages (whole coil, half coils, etc.) and the time derivative of the magnet excitation current. These signals are digitized and analyzed by the Digital Quench Detection (DQD) scan [3]. Upon detection of quench, both hardware (quench logic module) and software elements (all registered observers of the quench event) are notified. For safety reasons, the functionality of the DQD system is duplicated by analog quench detection circuitry. The DQD system can also be set up to send events when significant variations in monitored signals are seen, but not big enough to be identified as a quench. These events 


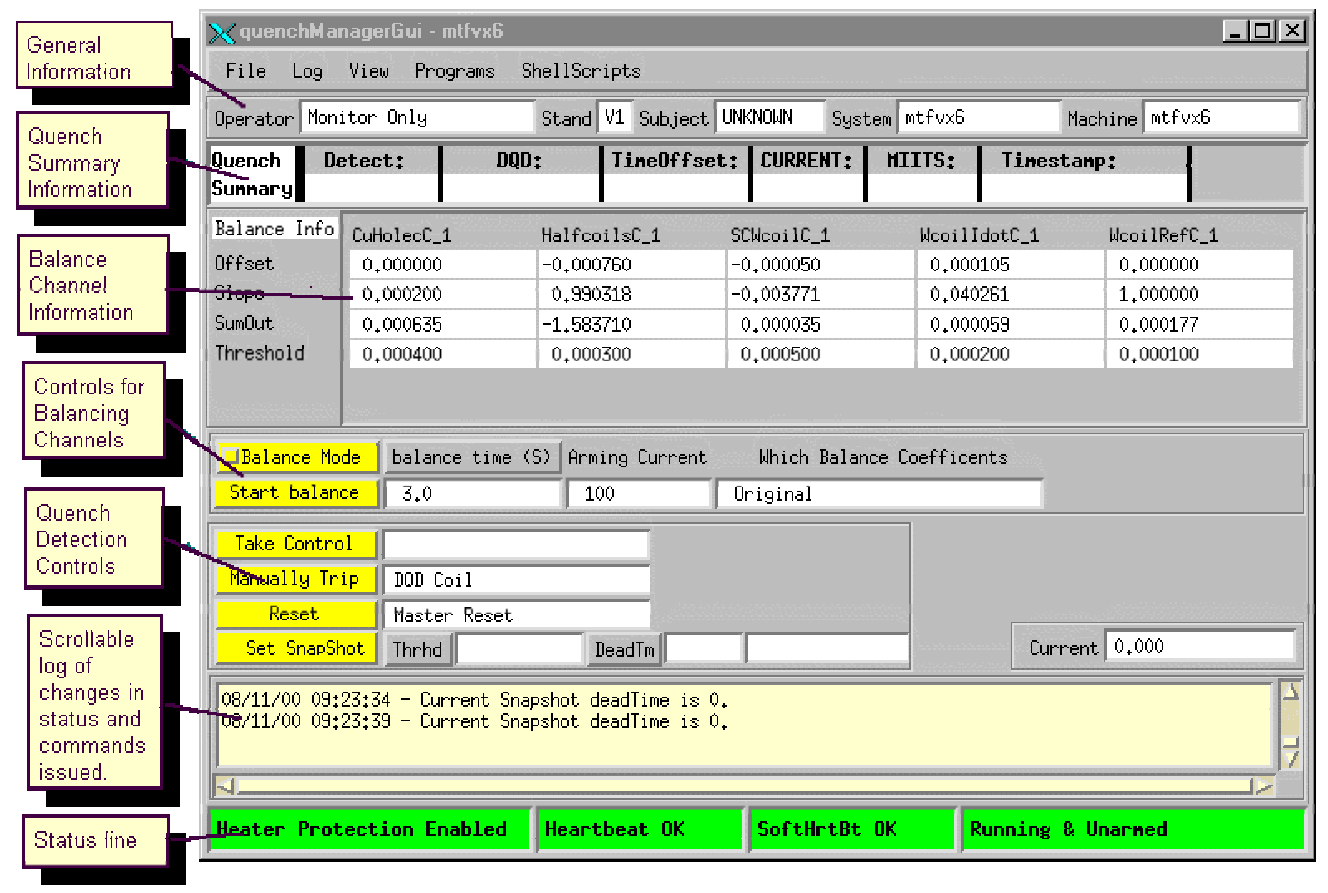

Figure 2: Main quench panel.

trigger the quench snapshot scan which archives quench detection data. This functionality does not impair the system's ability to react on quenches and store quench data.

The integrity of the quench detection system is continuously verified through the heartbeat signal. A missing heartbeat signal causes actions similar to a quench.

\subsection{Quench Protection}

Typical actions after detecting a quench include phasing back the power supply, firing the quench protection heaters, and activating the energy extraction dump resistor. These actions are conducted in hardware.

The heater firing units and the dump resistor are continuously monitored by the HFU-DUMP scan. The same scan also performs requested control actions (fire or charge heaters) and requested parameter updates (set delays, charge voltage, heater configurations).

The user is provided with GUIs to set up heater-firing units and dump parameters as well as to view their statuses.

\subsection{Quench Characterization}

Most of the information that is used to analyze quenches is collected by data loggers. The use of data loggers is dictated by relatively high data acquisition speeds (circa 10,000 samples per second). Data loggers are triggered by the hardware quench signal from the quench logic module. The functionality necessary to set up and control data loggers is provided by the data logger scan. The data logger scan, as any other scan, acquires its initial configuration from the RTDB and can be modified later on via the logger GUI that also shows the current logger state.

Apart from high rate data acquisition triggered by the quench signal, continuous monitoring of voltage tap signals and other channels is possible with slower rates (up to 10 samples per second). Monitored values can be visualized numerically or plotted versus time by appropriate GUIs.

\subsection{Power Supply System}

Integrated with the quench system is the power supply control system, which enables the user to control and monitor magnet power supplies. The central point of the system is the power server, a process that mediates between clients and the power supply controller [4]. The server works as a gateway that translates the client protocol into the controller protocol. It is also responsible for allocating control to only one client.

The power system can be controlled from a GUI, from within applications (via a special API), and from scripts (via a set of programs). Single ramp requests can be issued using any of these methods. In addition, ramp profiles can be loaded into the controller, including the saw tooth profile used for balancing quench detection circuits.

\subsection{Quench Management}

In addition to specialized user interfaces that focus on selected elements or subsystems (the power supply, data loggers, HFU, and dump interfaces), the user is provided with a set of GUIs to control and monitor the entire quench system.

Overall control of the quench system is performed with help of the main quench panel (see Fig. 2) that shows, 
among others, the current status of the system, summary data from quench analysis, and the recorded magnet current.

The exchange of software events can be monitored with the event monitor GUI that enables continuous monitoring of events or latching of event sequences.

Similar functionality, but for the hardware signals, is provided by the Change Of State (COS) GUI. With this tool the user can analyze the sequence of hardware state changes recorded by the COS scan.

The status of COS and other scans can be monitored and controlled via the scan panel GUI. Information such as error status, last run time, last error time, type of scan, and activity status can be viewed.

\section{QUENCH DATA}

Quench-related data is archived using the Chunk Format Standard (CFS) [5].

CFS provides a method of saving diverse formatted data in a file. Each file holds a hierarchical selection of related objects (chunks), while each chunk holds any number of data records of a specific type. Each chunk also contains a header with information about the chunk such as its type, size, version, and timestamp. The quench system utilizes a set of defined chunk formats, each pertaining to a different type of data. The structure of a typical quench file is shown in Fig. 3.

CFS supports distributed data archiving by transparently handling file locking and not enforcing any specific sequence of top-level chunks.

This type of a flexible and extensible data format is especially attractive for storing quench data, where various data acquired by different components and requiring different formats have to be stored together. Also, selective access to information is provided.

To facilitate analysis of quench data, an interactive graphical tool has been developed to visualize, transform, and compare recorded signals.

\section{SUMMARY}

A complete system to manage quenches of accelerator magnets in the R\&D environment has been developed. The system is built on top of an architectural framework, which defines structural elements of the system and specifies their interrelationships. The framework guarantees extensibility and flexibility of the quench management system. Such an approach allows also for easy integration with the rest of the test system and therefore straightforward synchronization and exchange of data.

A mixed hardware-software approach has been taken to quench detection. As a result, the system possesses superior performance and is characterized by improved monitoring of the quench detection process.

The system is also uniquely capable of slow monitoring and fast data logging of the same signals.

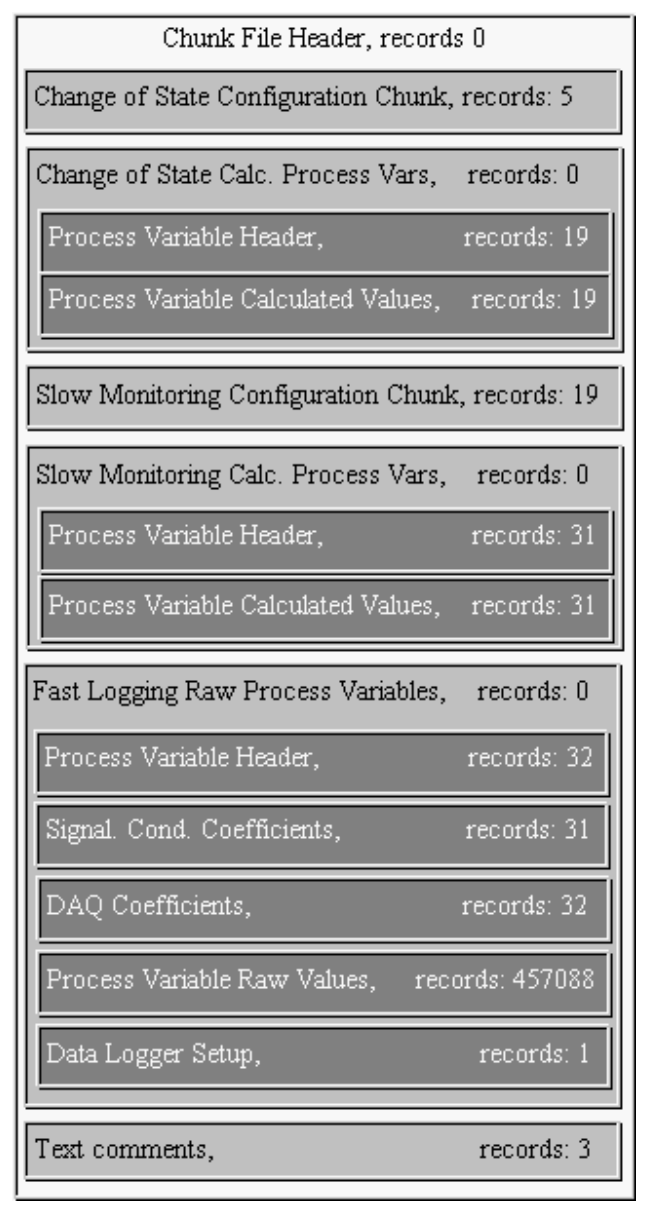

Figure 3: Quench file structure.

A complete state of the system is recorded for each quench, including diverse data coming from different, distributed sources.

The system, with its several GUIs, is user-friendly and relatively easy to manage. Also, its specialized tools simplify quench analysis.

\section{REFERENCES}

[1] J.M.Nogiec, E.Desavouret, D.Orris, J.Pachnik, S.Sharonov, J.Sim, J.C.Tompkins, and K.TromblyFreytag, "A Distributed Monitoring and Control System", PAC'97, Vancouver, (1997)

[2] J.M.Nogiec, E.Desavouret, J.Pachnik, S.Sharonov, and J.Sim, "An Open Distributed Monitoring and Control System", CHEP'97, Berlin, (1997)

[3] D.F. Orris, S. Feher, M.J. Lamm, J. Nogiec, S. Sharonov, M. Tartaglia, and J. Tompkins, "Digital Quench Detection System for Superconducting Magnets", PAC'99, New York, (1999)

[4] S.Sharonov, J.M.Nogiec, "An Embedded Power

Supply Controller", Pac'97, Vancouver, (1997)

[5] http://sdsg.fnal.gov/dmcsweb 